\title{
1 Cold shock induces a terminal investment reproductive response in $C$. elegans
}

3 Leah Gulyas ${ }^{1,2}$ and Jennifer R. Powell ${ }^{*}$

$5 \quad{ }^{*}$ Correspondence: jpowell@gettysburg.edu

$6 \quad{ }^{1}$ Department of Biology, Gettysburg College, Gettysburg, PA 17325 USA

$7 \quad 2$ Present Address: Plant and Microbial Biology Department, University of California

8 Berkeley, Berkeley, CA 94702, USA

11 Abstract

13 Challenges from environmental stressors have a profound impact on many life-history

14 traits of an organism, including reproductive strategy. Examples across multiple taxa have

15 demonstrated that maternal reproductive investment resulting from stress can improve

16 offspring survival; a form of matricidal provisioning when death appears imminent is

17 known as terminal investment. Here we report a reproductive response in the nematode

18 Caenorhabditis elegans upon exposure to acute cold shock at $2^{\circ} \mathrm{C}$, whereby vitellogenic lipid

19 movement from the soma to the germline appears to be massively upregulated at the

20 expense of parental survival. This response is dependent on functional TAX-2;TAX-4 cGMP-

21 gated channels that are part of canonical thermosensory mechanisms in worms and can be

22 prevented in the presence of activated SKN-1/Nrf2, the master stress regulator. Increased

23 maternal provisioning promotes improved embryonic cold shock survival, which is notably 
24 suppressed in animals with impaired vitellogenesis. These findings suggest that cold shock

25 in C. elegans triggers terminal investment to promote progeny fitness at the expense of

26 parental survival and may serve as a tractable model for future studies of stress-induced

27 progeny plasticity.

\section{Introduction}

31 Environmental stressors can severely jeopardize the ability of an organism and its progeny

32 to survive and reproduce, endangering organismal fitness. Consequently, organisms must

33 launch effective stress response mechanisms to mitigate damage, promote recovery,

34 and/or ensure progeny survival. A traditional view of life-history trade-offs assumes that

35 challenge from a stressor should invoke a response in which resources are shuttled away

36 from reproduction to support organismal defense and repair [1]. While this approach

37 temporarily decreases reproductive fitness, improved long-term survival may allow for

38 greater reproduction post-recovery. However, in a stressful environment where survival is

39 perceived to be unlikely, an alternate strategy may instead be to funnel resources into

40 reproduction, ensuring maximal output prior to parental death and subsequent survival of

41 the F1 generation [1].

43 The benefit of this strategy has been demonstrated in a number of organisms in different

44 environmental conditions [2]. Both biotic (i.e. pathogen exposure) and abiotic (thermal,

45 osmotic) stress conditions may potentiate molecular changes to reproduction. Alterations

46 ranging from epigenetic modifications to simple maternal cytosolic investments of 
47 macromolecules and nucleic acids have been associated with increased offspring resilience

48 to ensuing threats $[2,3]$. Yet despite myriad examples, the precise mechanisms by which

49 stressors induce these responses are still widely under exploration.

51 The nematode Caenhorhabditis elegans recently arose as an ideal model for investigating

52 stress-induced parental progeny investment. Several studies have detailed molecular

53 tactics by which worms modify and provision gametes and embryos in response to dietary

54 restriction, infection by pathogenic pseudomonads and microsporidia, and osmotic stress

55 [4-7]. Among an array of environmentally-relevant stressors, though, the reproductive

56 impacts of thermal stress (specifically cold stress) on C. elegans have not been closely

57 assessed. Both flies and mice employ reproductive strategies to respond to cold, with flies

58 inducing a more general stress response of reproductive dormancy and mice depositing

59 epigenetic modifications in sperm to promote retention of brown adipose tissue favoring

60 increased metabolic rates in offspring [8,9]. Given the likelihood of C. elegans regularly

61 encountering diurnal and seasonal temperature fluctuations, it seems probable that worms

62 may also have evolved rigorous reproductive programs to deal with cold exposure.

64 Several aspects of cold response in C. elegans have been well-characterized in the

65 literature, including thermotaxis and habituation [10-12]. Neurons and other tissues rely

66 on a multitude of signaling mechanisms for these physiological responses to occur, which

67 are in turn supported by a bevy of channels and other proteins. These processes are

68 described in detail in a recent review from Takeishi et al. [12]. Only lately, though, have

69 links between cold and reproduction begun to emerge. Sonoda et al. [13] demonstrate that 
70 the presence and integrity of sperm is required for normal cold tolerance by exerting an

71 effect through multiple tissues, including ASJ neuronal activity. At the level of population

72 dynamics, it has also been reported that $C$. elegans warming from a 2-hour cold exposure

73 enter a state of programmed death which is thought to result in kin selection for the

74 survival of younger (and presumably more reproductively fit) animals [14]. However, the

75 extent to which acute cold stress directly impacts reproductive capacity in these animals

76 remains unknown. As alternating periods of cold and moderate temperatures preceding a

77 winter season may comprise a substantial portion of the short reproductive window in $C$.

78 elegans, this necessitates further study.

80 We have previously shown that acute cold shock at $2^{\circ} \mathrm{C}$ causes loss of intestinal

81 pigmentation, immobility, and reproductive disruption in wild-type hermaphrodite worms.

82 In many cases, this phenotypic program results in lethality [15]. Here we extend this work

83 by characterizing the induction of these phenotypes via the neuronal TAX-2/TAX-4

84 thermotransduction channel, mediated by the canonical stress response regulator SKN-

85 1/Nrf2. We further describe a role for the normal vitellogenesis machinery in reallocating

86 pigmented intestinal lipid supplies to the germline, which appears to come at the expense

87 of parental survival following cold exposure. Overall, our findings suggest that acute cold

88 shock provokes a terminal investment reproductive response in C. elegans, making this

89 discovery an exciting opportunity to explore the phenomenon in a genetically tractable

90 system.

91

\section{Results}




\section{Acute cold shock causes drastic phenotypic alterations}

95 The duration of cold exposure for young adult hermaphrodite C. elegans at $2{ }^{\circ} \mathrm{C}$ is negatively

96 correlated to post-shock survival rates [15]. Wild-type hermaphrodite worms exposed to a

97 4-hour cold shock (CS) do not initially display high mortality rates (Fig. 1a); this allows

98 observation of a range of phenotypic transitions as they recover from the limited-duration

99 cold stress at their preferred temperature of $20^{\circ} \mathrm{C}$. One of the most striking phenotypes

100 exhibited in post-cold shock (post-CS) animals during the recovery period is a dramatic

101 decrease in pigmentation in the normally highly pigmented intestine, so that the body

102 becomes almost entirely clear (Fig. 1b, c) [15]. This is often accompanied by motor and

103 reproductive disruptions such as mobility loss, withering of the gonad arms, decreased

104 number of internal embryos, and the eventual death of about 30\% of the population (Fig.

105 1a-d) [15]. It should be noted that these phenotypic responses do not appear to be due to

106 any relative heat shock following the transition from $2^{\circ} \mathrm{C}$ to $20^{\circ} \mathrm{C}$ as the expression of GFP-

107 tagged HSP-4 (heat shock protein) is not induced following cold shock (Fig.

108 1e). Interestingly, some CS wild-type animals regain pigmentation after clearing; these

109 worms do not die and display a general reversal of the other negative impacts of cold shock

110 (Fig. 1b)[15]. We sought to better understand the factors regulating the post-CS recovery

111 program in wild-type worms, focusing particularly on the functional role of pigmentation

112 loss and the genetic components involved in producing it. 

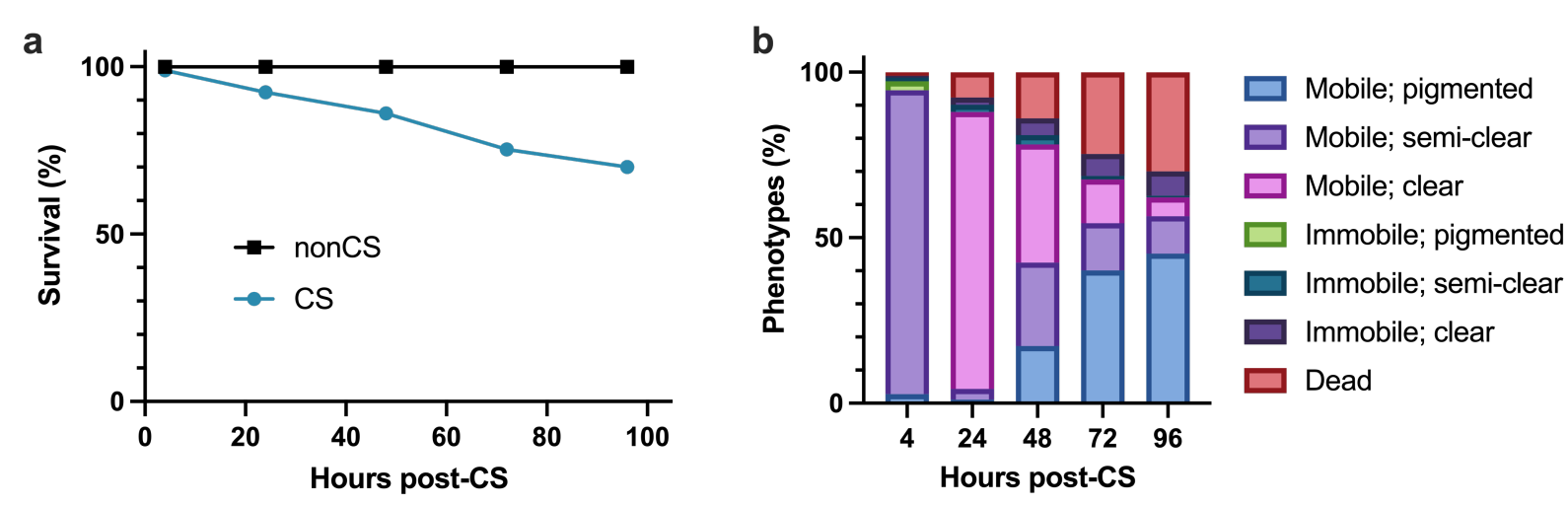

C

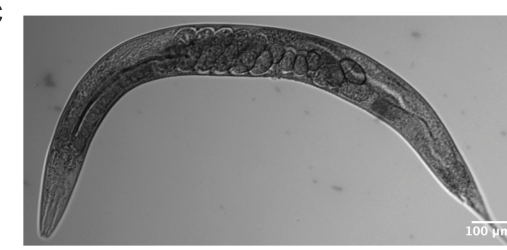
N2 noncs

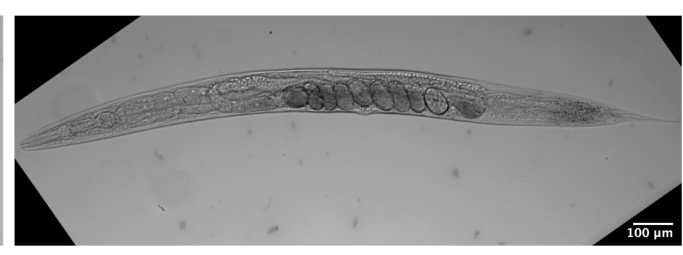

N2 CS
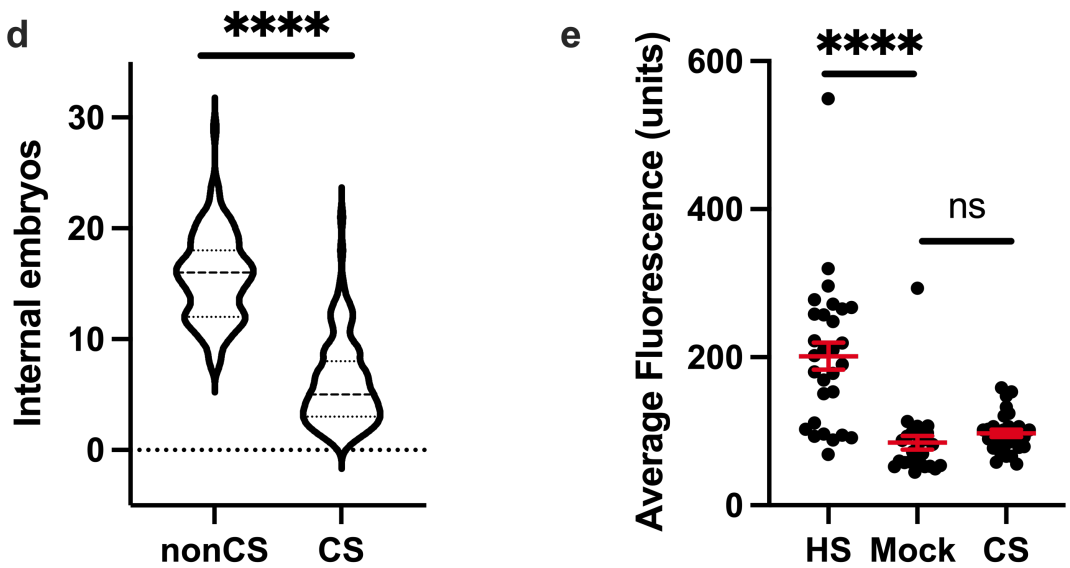

Figure 1. Cold-shocked worms show decrease in survival and characteristic phenotypic alterations. $\mathrm{N} 2$ young adult hermaphrodites were shifted from $20^{\circ} \mathrm{C}$ to $2^{\circ} \mathrm{C}$ for a $4 \mathrm{~h}$ cold shock (CS) and thereafter recovered at $20^{\circ} \mathrm{C}$ for $96 \mathrm{~h}$ with assessment of (a) survival and (b) phenotypic alterations ( $\mathrm{n}$ =177). Death and immobility were assayed by nose tap; worms were considered to be immobile if the tap elicited slight movement in the head region but no other body movement, and dead worms were completely unresponsive. (c) Representative images of young adult N2 hermaphrodites following cold shock. (d) Average number of internal embryos per worm in CS versus nonCS conditions (Mann-Whitney $\mathrm{U}$ test: $\mathrm{U}=604$, two-tailed $\mathrm{P}<0.0001$ ). (e) Induction of heat shock protein HSP-4::GFP 12 hours after either $2 \mathrm{~h}$ heat shock at $35^{\circ} \mathrm{C}, 4 \mathrm{~h}$ cold shock at $2^{\circ} \mathrm{C}$, or no treatment $(\mathrm{n} \geq 26$ per condition; Kruskal-Wallis test: $\mathrm{H}=33.86$, $\mathrm{P}<0.0001$; Dunn's Multiple Comparison Test: ${ }^{* * * *} \mathrm{P}<0.0001$ ). Error bars are mean \pm s.e.m. 
115 Since pigmentation in the $C$. elegans intestine is due in part to the presence of lipid-storing

116 fat droplets [16], we wondered whether the decrease in pigmentation in cold-shocked

117 worms corresponds to a depletion of intestinal lipid supplies, potentially as a result of

118 increased metabolism meant to fuel post-cold shock recovery. Using Nile Red, which

119 accumulates and fluoresces in hydrophobic environments [17], as an indicator of lipid

120 content, we therefore analyzed the fat stores of worms 12 hours following CS (note that all

121 cold shocks were performed for $4 \mathrm{~h}$ at $\left.2^{\circ} \mathrm{C}\right)$. We indeed observed a significant decrease $(\mathrm{P}<$

122 0.0001) in the average lipid content per worm (Fig. 2a); visually, this presents as an overall

123 reduction in average fluorescence that is most striking in the intestine (Fig. 2c). However,

124 we also unexpectedly noted that the lipid content of the gonad appears to concomitantly

125 increase, marked by the presence of fluorescent (and therefore lipid-rich) internal embryos

126 in the gonads of many cold-shocked worms that were mostly absent in their non-cold

127 shocked counterparts (Fig. 2b, c). These embryos are somewhat sporadic, usually

128 accounting only for a proportion of all embryos in the germline and are interspersed with

129 non-fluorescent embryos. Importantly, the percentage of fluorescent internal embryos per

130 worm was found to be elevated two-fold in CS worms relative to that of nonCS controls

131 (Fig. 2b). These observations suggest that rather than just metabolically depleting lipid

132 supplies, C. elegans may also reallocate lipids from the intestine to the germline. To further

133 analyze this process spatiotemporally, we performed a time course of Nile Red staining

134 following cold shock (Fig. 2c). Upon entry into $2^{\circ} \mathrm{C}$ shock, young adult worms enter a "chill

135 coma," ceasing virtually all movement $[15,18]$. Following exit from cold shock, most worms

136 gradually resume movement over the first 30 minutes of recovery at $20^{\circ} \mathrm{C}$. At this point, the

137 distribution of Nile Red-staining lipids is indistinguishable from non-cold shocked controls, 
138 indicating that lipid reallocation occurs after, rather than during, the cold shock. By 4 hours

139 post-cold shock, we observed visually decreased but rarely absent fluorescence in the

140 intestine; at this time, many worms have brightly fluorescing proximal oocytes, suggesting

141 that lipids are beginning to relocate from the intestine into the germline. This process of

142 reallocation appears to continue over the next $14+$ hours, with many 12 h-recovered

143 worms containing fluorescent embryos and oocytes but only slight intestinal fluorescence,

144 and most 18 h-recovered worms fluorescing almost exclusively in the embryos. Though it

145 is unclear what other factors (e.g. metabolism) may contribute to the overall pigmentation

146 loss, these observations suggest that the dramatic decrease in intestinal pigmentation

147 results in large part from the reallocation of lipids from somatic tissues to the germline

148 during the recovery phase following a severe cold shock. 

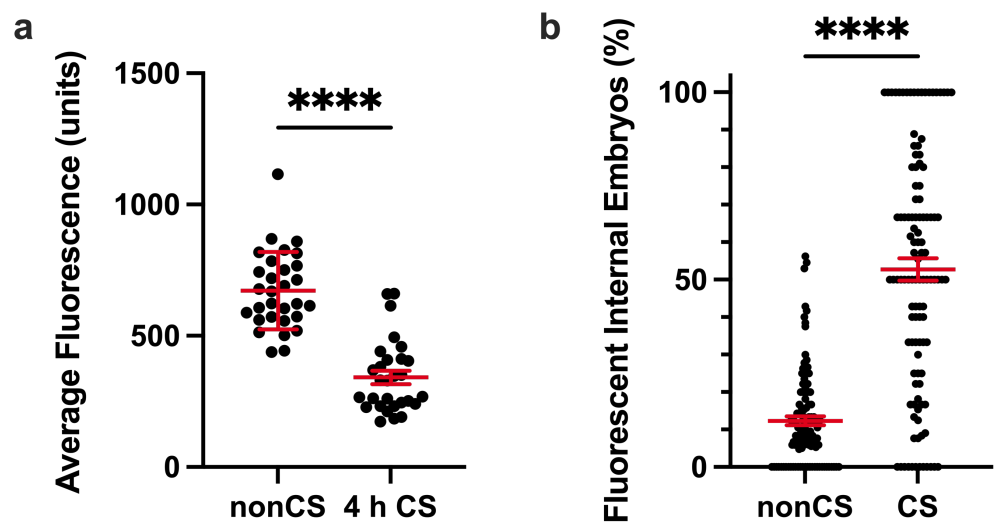

C

$\begin{array}{ll}\text { Recovery } & \text { nonCS control } \\ \text { Time } & \end{array}$

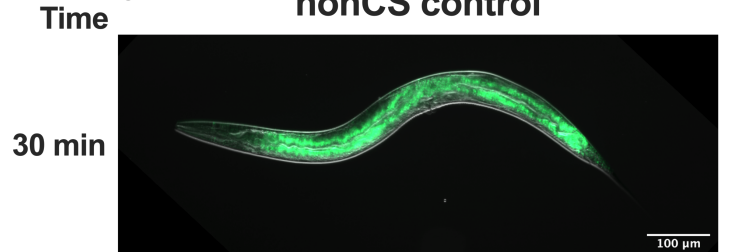

$4 \mathrm{~h}$

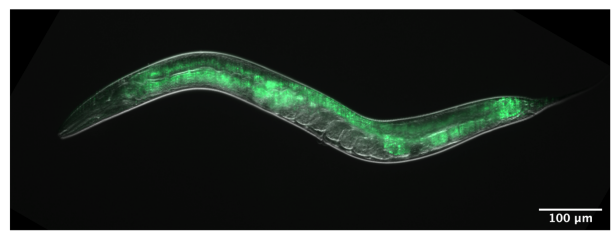

$12 \mathrm{~h}$

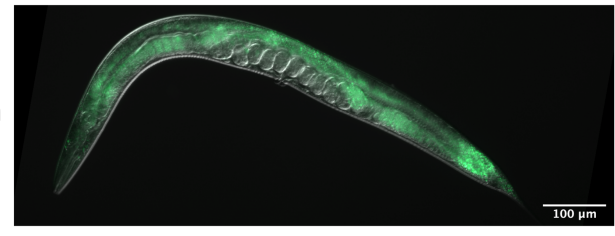

$18 \mathrm{~h}$

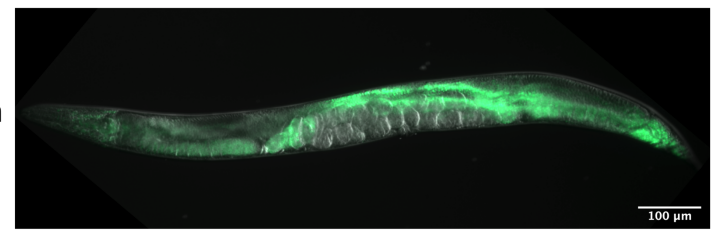

$4 \mathrm{~h} C S$
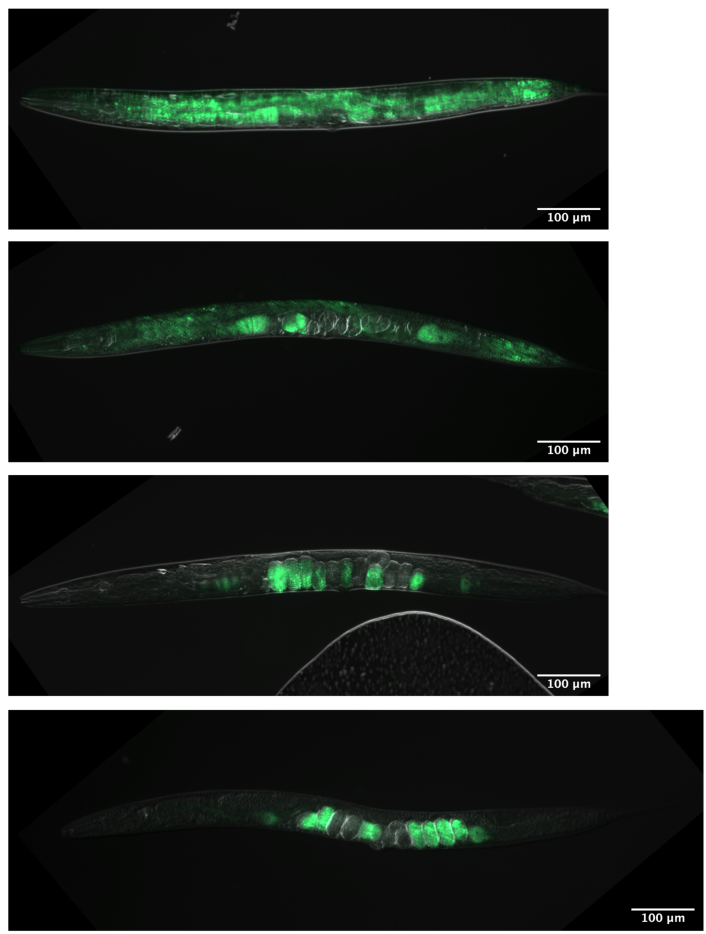

Figure 2. Cold shock recovery is associated with lipid localization shifts from the intestine to the germline. Young adult $\mathrm{N} 2$ hermaphrodites were exposed to $2^{\circ} \mathrm{C}$ cold shock or control nonCS conditions $\left(20^{\circ} \mathrm{C}\right)$ for $4 \mathrm{~h}$ and recovered before Nile Red staining and imaging for lipid content. (a) Average fluorescence per worm ( $\mathrm{n} \geq 29$ per condition; Mann-Whitney $U$ test: $U=48$; two-tailed $P<0.0001)$ and (b) percent of internal fluorescent embryos per worm $(n \geq 111$; Mann-Whitney $U$ test: $U=1885$; two-tailed $P<$ 0.0001 ) were quantified $12 \mathrm{~h}$ post-CS. Error bars are mean \pm s.e.m. (c) Representative images of Nile Redstained worms at indicated time points post-CS show progression of intestinal lipid loss and relocalization to the embryos. 


\section{Thermosensation is required to initiate the cold shock response}

151 We next asked how wild-type $C$. elegans perceive and interpret cold shock as a signal for

152 lipid reallocation. The cGMP-gated channel subunits TAX-2 and TAX-4 are important for

153 thermosensation and acquired cold tolerance in worms, acting specifically in the ASJ

154 neurons $[11,19,20]$. We reasoned that worms might also rely on a neuronal mechanism of

155 sensation that acts through the TAX-2/TAX-4 channel to induce the cold shock response in

156 the absence of prior cold exposure. tax-2(p671); tax-4(p678) double loss-of-function

157 mutants were therefore tested for sensitivity to cold shock. Not only do these mutants

158 show 100\% survival following cold shock (Fig. 3a), but, unlike wild-type worms, they also

159 do not display any significant difference (P > 0.9999) in overall lipid content $12 \mathrm{~h}$ after CS,

160 nor the striking lipid reallocation phenotype characterized by large numbers of fluorescent

161 embryos (Fig. 3b-d). Interestingly, despite this resilience to shock, $\operatorname{tax}-2$ (p671); tax-

$1624(p 678)$ mutants still show decreased internal embryo quantities, though not to the same

163 degree as N2 worms (Fig. 3e), suggesting that cold exposure has some additional impact on

164 fertility independent of this thermosensation pathway. Taken together, though, these data

165 support the hypothesis that fat reallocation as a stress response following CS may depend

166 on a neuronal mechanism for induction. Since TAX-2/TAX-4 plays a role in temperature

167 sensation, we speculate that canonical cold thermosensation is required for lipid

168 reallocation to take place. 
bioRxiv preprint doi: https://doi_org/10 1101/2021.09 11.459896; this version posted September 11, 2021. The copyright holder for this preprint (which was not certified by peer review) is the author/funder. All rights reserved. No reuse allowed without permission.
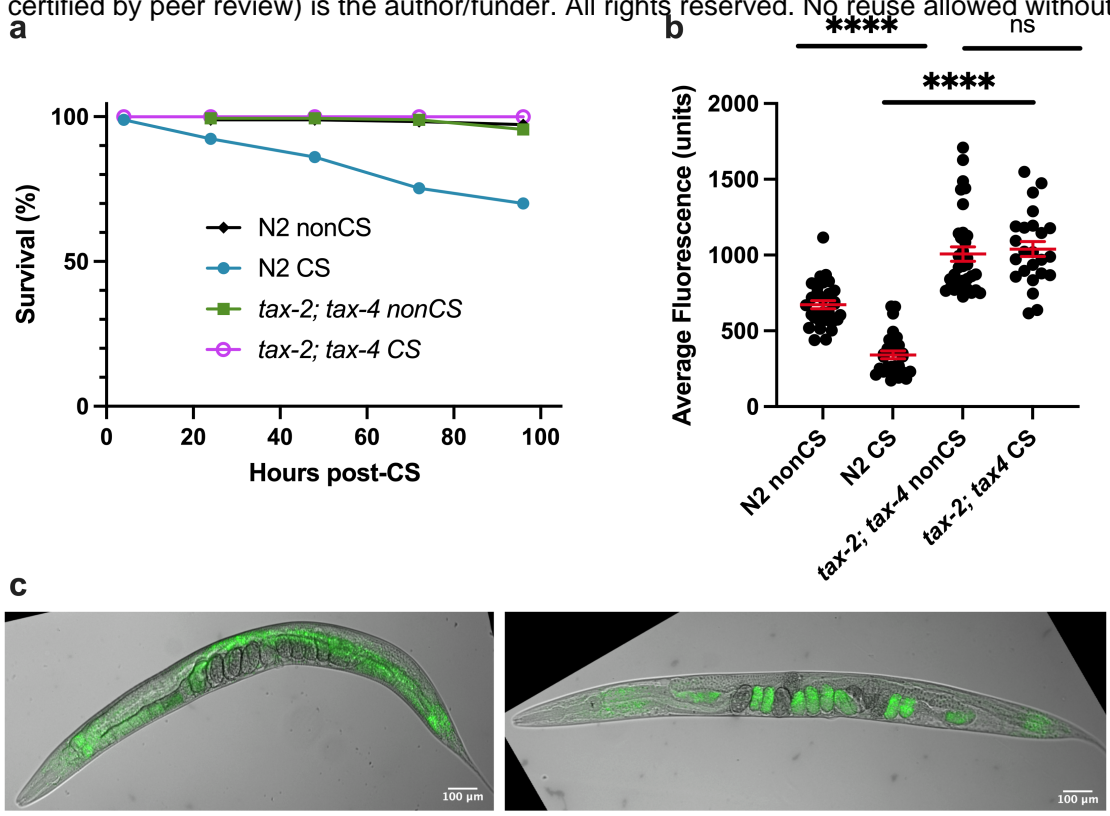

N2 nonCS N2 CS
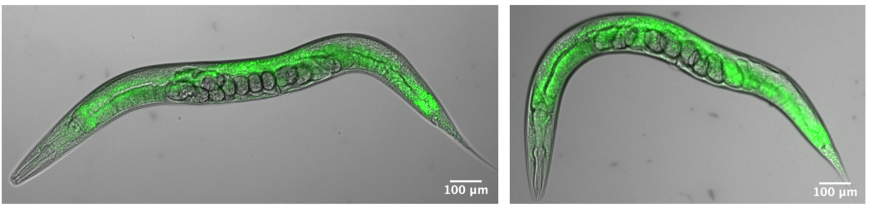

$\operatorname{tax}-2 ; \operatorname{tax}-4$ nonCS

tax-2; tax-4 CS

d

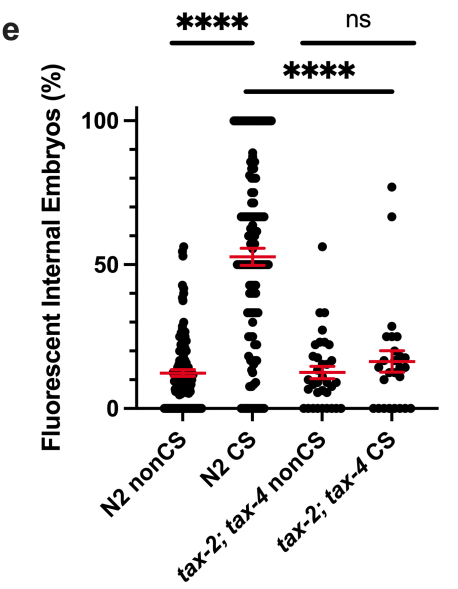

Figure 3. TAX-2; TAX-4-mediated thermosensation is required for lipid relocalization following cold shock. (a) tax-2(p671); tax-4(p678) double loss-of-function mutants were cold shocked and recovered while monitoring survival rates ( $\mathrm{n} \geq 120$ worms per condition). (b) At $12 \mathrm{~h}$ post-CS or nonCS control, tax-2(p671); tax-4(p678) were Nile Red lipid-stained and the average fluorescence per worm was quantified (Kruskal-Wallis test: $\mathrm{H}=83.83, \mathrm{P}<0.0001$; Dunn's Multiple Comparison test: ${ }^{* * * *} \mathrm{P}<$ 0.0001). (c) Representative images of Nile Red staining show retention of fluorescence in the intestines of tax-2; tax-4 mutants. (d) Number of embryos (Kruskal-Wallis test: $\mathrm{H}=171.1, \mathrm{P}<0.0001$; Dunn's Multiple Comparison test: ${ }^{*} \mathrm{P}=0.0484,{ }^{* * * *} \mathrm{P}<0.0001$ ) and (e) percent fluorescent internal embryos (KruskalWallis test: $\mathrm{H}=100.1, \mathrm{P}<0.0001$; Dunn's Multiple Comparison test: ${ }^{* * * *} \mathrm{P}<0.0001$ ) were quantified per worm from Nile Red images ( $n \geq 24$ worms per condition for b-e; error bars are mean \pm s.e.m.). 
171 After determining a requirement for neuronal signaling in cold-shock-induced lipid

172 reallocation, we next wondered what intermediate factors were needed to translate the

173 cold stimulus into a signal to mobilize resources. The master regulatory transcription

174 factor SKN-1/Nrf2 coordinates a return to homeostasis following a variety of stresses,

175 including oxidative stress [4], pathogen infection [21,22], and osmotic stress [23]. We

176 therefore predicted that skn-1 would perform a similar function during cold stress

177 recovery. Indeed, skn-1(lax188) gain-of-function mutants [24] are highly resistant to cold

178 shock, with nearly $100 \%$ survival rates 96 host-CS (Fig. 4a). Consistent with a retention of

179 overall lipid stores by Nile Red Staining (Fig. 4b,d), these mutants did not contain

180 significantly greater numbers of fluorescing internal embryos following cold shock (P >

181 0.9999), though they did have a marginal reduction in the number of internal embryos

182 (Fig. 4d-f). Conversely, skn-1(mg570) loss-of-function mutants displayed a wild-type cold

183 shock response, with significant reallocation $(\mathrm{P}<0.00001)$ of lipids to the germline and

184 loss of somatic fats (Fig. 4c-f). These data suggest that the $s k n-1 a$ isoform, which is knocked

185 out by the $\mathrm{mg} 570$ mutation [25], is not required for lipid mobilization in response to cold

186 shock. However, SKN-1 activation protects cold shocked worms from recovery phase

187 lethality and prevents lipid reallocation. 
bioRxiv preprint doi: https://doi.org/10.1101/2021.09.11.459896; this version posted September 11, 2021. The copyright holder for this preprint

a

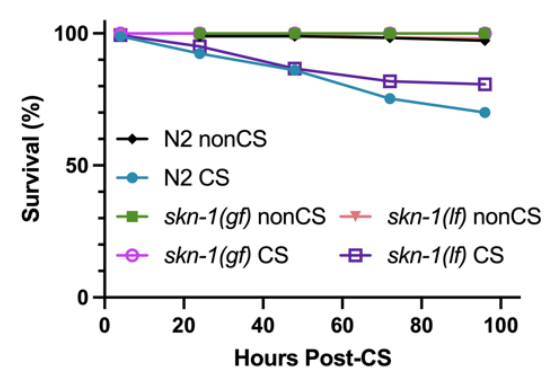

d
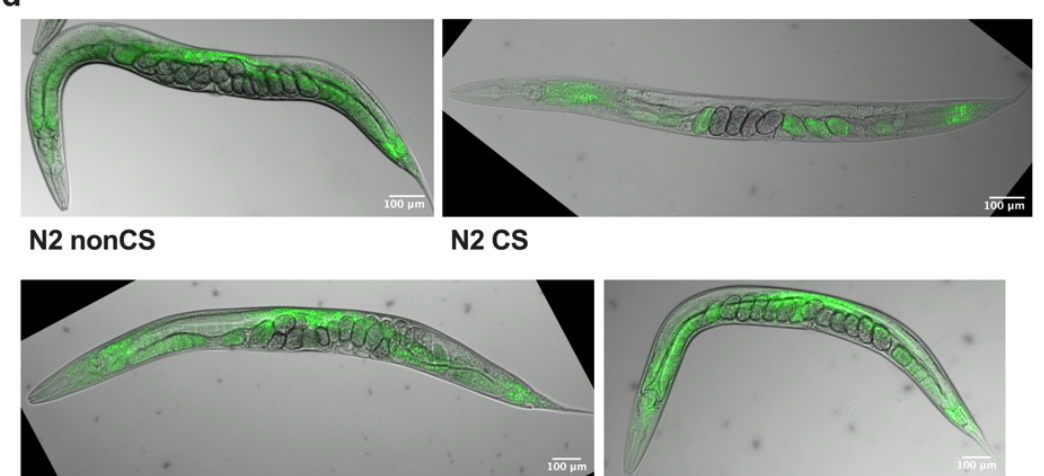

skn-1(gf) nonCS

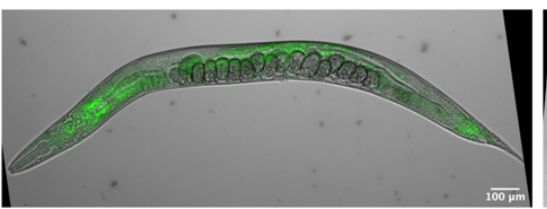

skn-1(If) nonCS

e

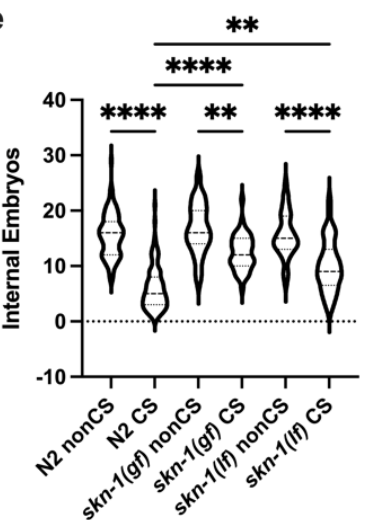

b

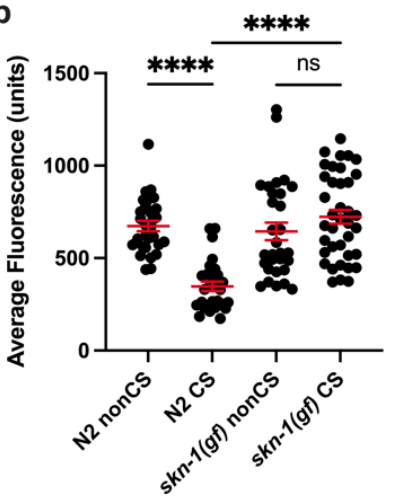

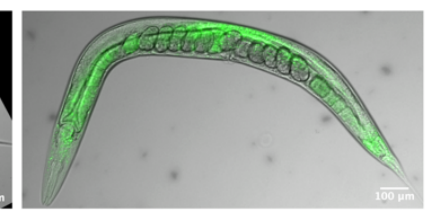

$s k n-1(g f)$ CS

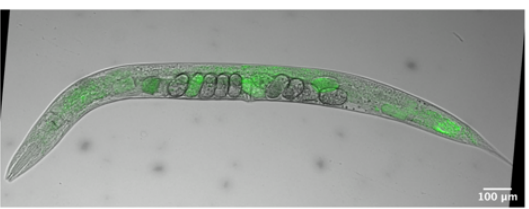

skn-1(If) CS

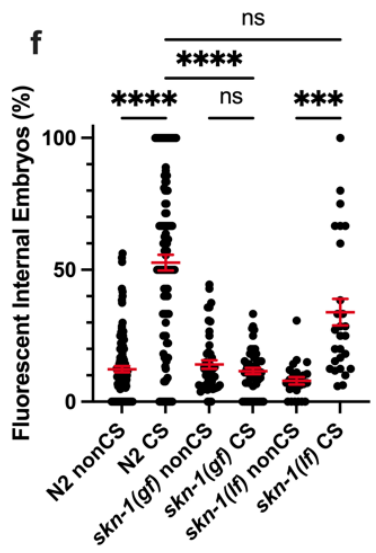

c

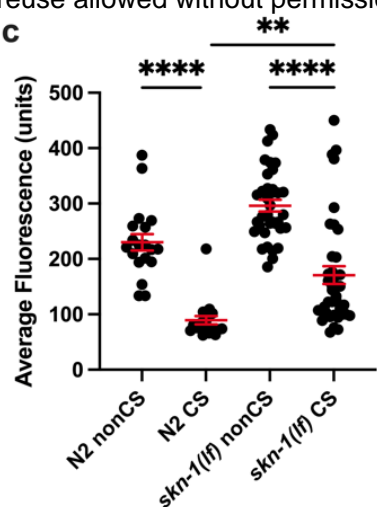

Figure 4. Activated SKN-1 prevents lipid relocalization following cold shock (a) skn-1(lax188) gain-offunction mutants and skn-1(mg570) loss-of-function mutants were cold shocked and recovered while monitoring survival rates ( $\mathrm{n} \geq 120$ worms per condition). (b) At $12 \mathrm{~h}$ post-CS or control nonCS, skn1(lax188) and skn-1(mg570) were Nile Red lipid-stained and average fluorescence per worm quantified (N2 v. skn-1(lax188)- Kruskal-Wallis test: $\mathrm{H}=47.46, \mathrm{P}<0.0001$; Dunn's Multiple Comparison test: ${ }^{* * * *} \mathrm{P}<$ 0.0001; N2 v. skn-1(mg570)- Kruskal-Wallis test: H=64.55, P < 0.0001; Dunn's Multiple Comparison test: 
${ }^{* *} \mathrm{P}=0.0064,{ }^{* * * *} \mathrm{P}<0.0001$ ). (c) Relative to wild-type N2s, representative images of $s k n-1$ (lax188) mutants show maintenance of intestinal fluorescence contrary to $s k n-1$ (mg570), which show lipid relocalization. (d) Number of embryos (Kruskal-Wallis test: $\mathrm{H}=223.1, \mathrm{P}<0.0001$; Dunn's Multiple Comparison test: ${ }^{* *} \mathrm{P}_{s k n-1(g f)}$ noncs vs. CS $\left.=0.0020,{ }^{* *} \mathrm{P}_{\mathrm{N} 2 \text { c } \operatorname{cs~vs.~} s k n-1(f) \text { CS }}=0.0014,{ }^{* * * *} \mathrm{P}<0.0001\right)$ and (e) percent of fluorescent internal embryos (Kruskal-Wallis test: $\mathrm{H}=132.0, \mathrm{P}<0.0001$; Dunn's Multiple Comparison test: $\left.{ }^{* * *} \mathrm{P}=0.0002,{ }^{* * * *} \mathrm{P}<0.0001\right)$ were quantified per worm from Nile Red images $(\mathrm{n} \geq 26$ worms per condition for b-e; error bars are mean \pm s.e.m.).

\section{Lipid reallocation results from upregulated vitellogenesis following cold shock}

190 The process governing the normal movement of lipids from the soma to the germline is

191 vitellogenesis, requiring the vitellogenin proteins coded for by vit-1-6 genes. Once coupled

192 to somatic lipid supplies, the resulting lipoprotein complexes shuttle to the germline

193 [26]. We hypothesized that the lipid mobilization induced by cold shock is a result of the

194 normal vitellogenesis machinery being commandeered as part of a stress response.

195 One of the major vitellogenins is VIT-2, which generates a $170 \mathrm{kD}$ yolk protein product

196 known as YP170B [27]. We predicted that loss of vit-2 would reduce lipid reallocation

197 following CS. Since preventing reallocation in our previous mutants also increased post-CS

198 survival, we expected that vit-2 loss of function would enhance protection from

199 CS. Consistent with these hypotheses, impairment of vitellogenesis in vit-2(ok3211) loss-of-

200 function mutants produced phenotypes similar to tax-2(p671); tax-4(p678) animals. In

201 addition to maintaining high survival rates, CS vit- 
a

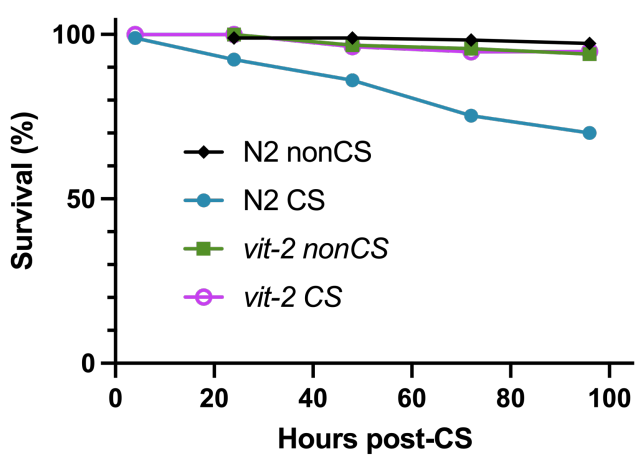

b

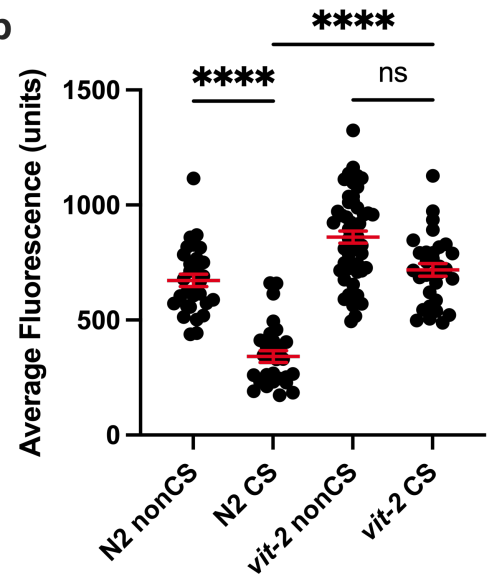

C

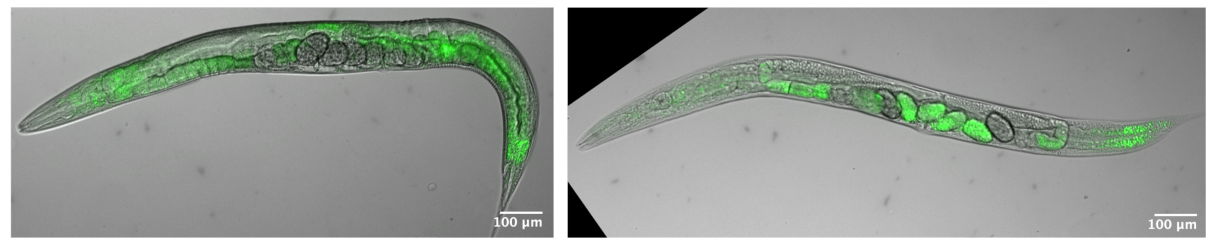

N2 nonCS

N2 CS

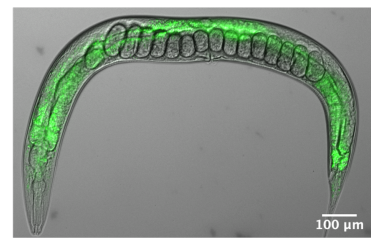

vit-2 nonCS

d

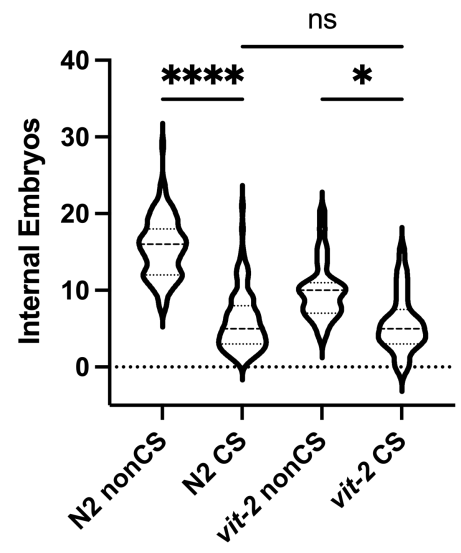

e

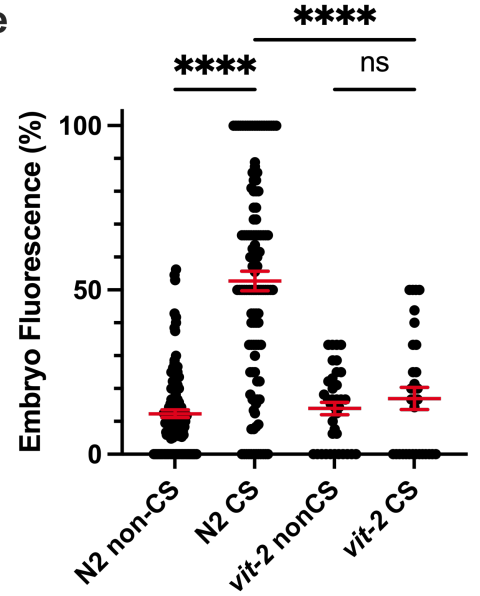

Figure 5. VIT-2-regulated vitellogenesis during recovery promotes lipid relocalization and impairs survival. (a) vit-2(ok3211) loss-of-function mutants were cold shocked and recovered while monitoring survival rates ( $\mathrm{n} \geq 130$ worms per condition). (b) At 12 h post-CS or nonCS, vit-2(ok3211) were Nile Red lipid-stained and average fluorescence per worm quantified ( $\mathrm{n} \geq 31$ worms per condition; Kruskal-Wallis test: $\mathrm{H}=75.18, \mathrm{P}<0.0001$; Dunn's Multiple Comparison test: $\left.{ }^{* * * *} \mathrm{P}<0.0001\right)$. Error bars are mean \pm s.e.m. (c) Images of Nile Red Staining show intestinal lipid retention in vit-2 mutants. (d) Number of embryos (Kruskal-Wallis test: $\mathrm{H}=169.9, \mathrm{P}<0.0001$; Dunn's Multiple Comparison test: ${ }^{*} \mathrm{P}=0.0149,{ }^{* * * *} \mathrm{P}<0.0001$ ) and (e) percent of fluorescent internal embryos (Kruskal-Wallis test: $\mathrm{H}=101.5, \mathrm{P}<0.0001$; Dunn's Multiple Comparison test: $\left.{ }^{* * * *} \mathrm{P}<0.0001\right)$ were quantified per worm from Nile Red images ( $\mathrm{n} \geq 31$ for $\mathrm{b}$-e; error bars are mean \pm s.e.m.). 
202 2(ok3211) mutants did not undergo a substantial loss of intestinal lipid supplies, nor did

203 they exhibit a marked increase in internal embryo fluorescence (despite a

204 modest decrease in internal embryo counts) (Fig 5a-e).

205 Since other vitellogenin transcripts produce different lipoprotein forms for movement to

206 the germline, we decided to additionally test whether a member of the YP170A-generating

207 class of vitellogenins would recapitulate our results with vit-2. To this end, we analyzed the

208 CS phenotypes of vit-5(ok3239) loss-of-function animals. Indeed, we found that as in vit-2

209 mutants, survival was rescued in these animals following CS, corresponding with a

210 retention of somatic lipids and inhibition of embryonic lipid reallocation (Fig. 6a-c,e). As

211 with other mutants that prevent lipid reallocation, there was still an effect of cold in

212 reducing overall embryo output $12 \mathrm{~h}$ post-CS, hinting that temperature impacts fertility

213 independent of the other reproductive alterations exhibited by wild-type worms (Fig. 6d). 
bioRxiv preprint doi: https://doi.org/10.1101/2021.09.11.459896; this version posted September 11, 2021. The copyright holder for this preprint (which was not certified by peer review) is the author/funder. All rights reserved. No reuse allowed without permission.
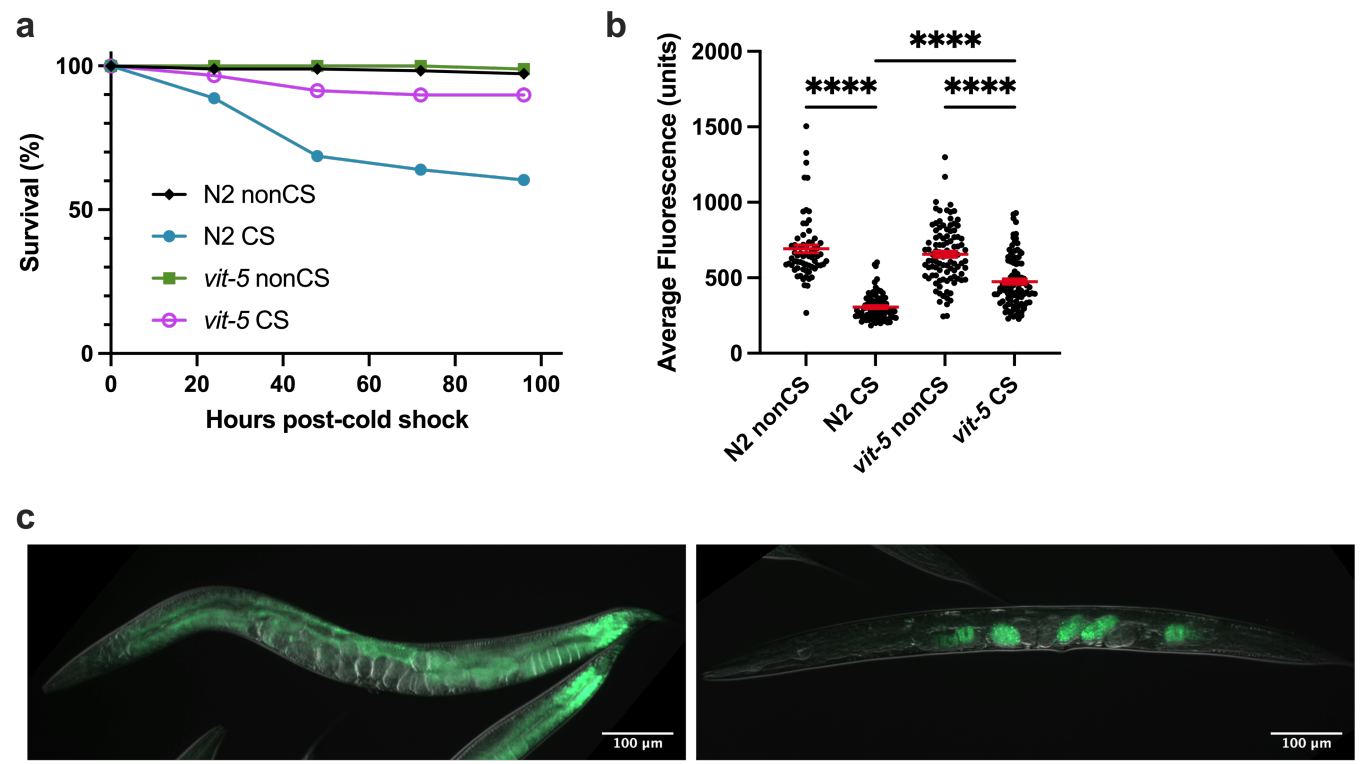

\section{N2 nonCS}

N2 CS
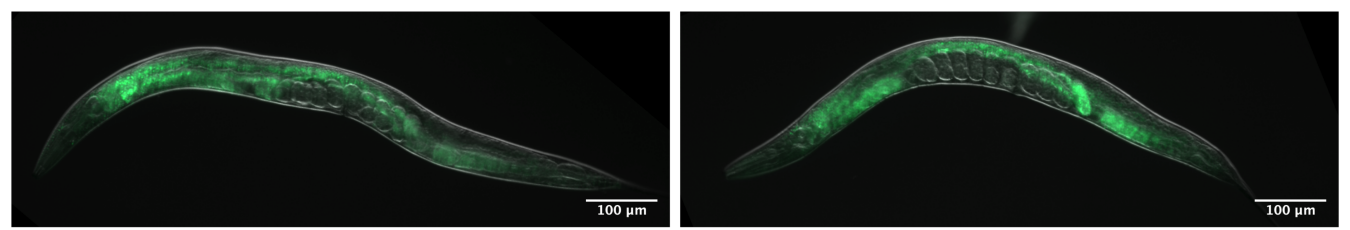

vit-5 nonCS

vit-5 CS
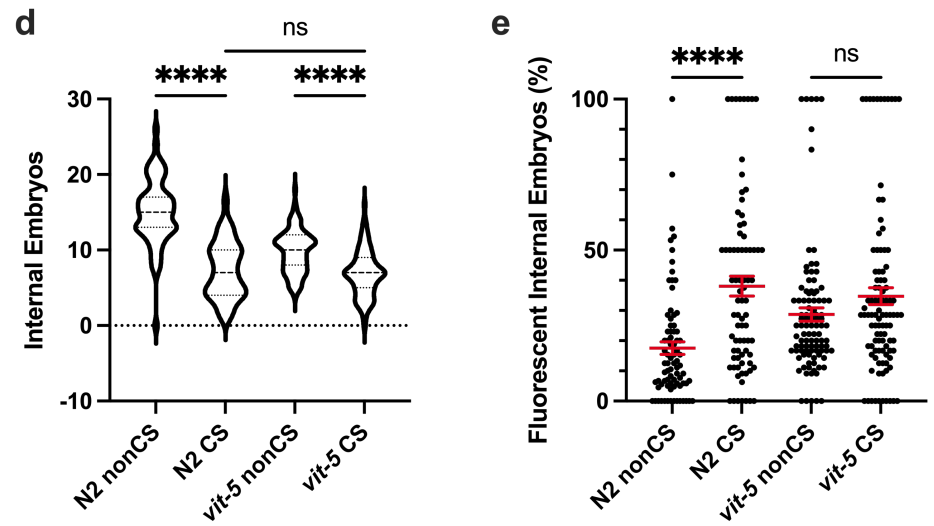

Figure 6. VIT-5 vitellogenin family also promotes embryo lipid reallocation during cold shock recovery. (a) vit-5(ok3239) loss-of-function mutants were cold shocked and recovered while monitoring survival rates ( $\mathrm{n} \geq 170$ worms per condition). At $12 \mathrm{~h}$ post-CS or control nonCS, vit-5(ok3239) were Nile Red lipid-stained and average fluorescence per worm quantified ( $\mathrm{n} \geq 67$ worms per condition; KruskalWallis test: $\mathrm{H}=172.0, \mathrm{P}<0.0001$; Dunn's Multiple Comparison test: ${ }^{* * * * *} \mathrm{P}<0.0001$. Error bars are mean \pm s.e.m. (c) Representative Nile Red staining shows intestinal fluorescence in vit-5(ok3239) following cold shock. (d) Number of embryos (Kruskal-Wallis test: $\mathrm{H}=137.2, \mathrm{P}<0.0001$; Dunn's Multiple Comparison test: ${ }^{* * * *} \mathrm{P}<0.0001$ ) and (e) percent of fluorescent internal embryos (Kruskal-Wallis test: $\mathrm{H}=37.58, \mathrm{P}<$ 0.0001; Dunn's Multiple Comparison test: $\mathrm{H}=37.58$, $\left.{ }^{* * * *} \mathrm{P}<0.0001\right)$ were quantified per worm from Nile Red images ( $\mathrm{n} \geq 67$ worms per condition; error bars are mean \pm s.e.m.). 


\section{Cold shock response is a form of a terminal investment}

217 Our data thus far suggest that upon recovery from acute CS, wild-type C. elegans induce a

218 reproductive phenotype whereby somatic lipid supplies are massively relocalized to the

219 germline using the normal vitellogenesis machinery. This appears to come at the expense

220 of the parent's own mortality, as retaining somatic lipids by preventing thermosensation or

221 vitellogenesis is sufficient to rescue survival. Such a tradeoff between survival and

222 reproduction is redolent of the terminal investment hypothesis (reviewed in Gulyas and

223 Powell, 2019 [2]), which predicts that in some instances of acute stress where the

224 likelihood of death is high, organisms can preferentially funnel resources to reproduction

225 to maximize reproductive fitness at the cost of survival.

226 To determine whether cold stress-associated phenotypes in C. elegans are an example of

227 such a process, we eliminated all potential for reproductive investment by assaying sterile

228 glp-1(e2141) and glp-1(q231) worms to ask whether these worms still exhibited lipid loss

229 or death upon cold shock. Strikingly, the absence of a germline in these animals completely

230 prevented both intestinal clearing and death, again confirming that lipid movement from

231 the soma to the germline is associated with parental lethality (Fig. 7a). If resource

232 reallocation to the progeny is indeed meant to increase reproductive fitness in inclement

233 conditions, there should be some benefit to the progeny of CS worms that offspring of

234 nonCS parents do not receive. We speculated that in the case of environmental CS, a

235 sudden, seasonal, cold to warm cycle might signal likelihood of future cold conditions that

236 would impede the ability of embryos to hatch and survive to reproductive age. We

237 therefore devised an assay to test for the relative fitness of embryos in cold conditions 
238 depending on whether they came from nonCS or CS parents and were thus more likely to

239 have received lipid provisioning. To do this, embryos were collected from nonCS and CS

240 parents within a $2 \mathrm{~h}$ time window when post-CS lipid reallocation seems to peak and

241 subsequently exposed them to a cold stress of 24 hours. We then assessed hatching rates

24224 hours following the embryonic cold shock. Excitingly, embryos coming from parents

243 that had experienced cold shock prior to reproduction exhibited a small but significant

244 increase in hatching rates relative to their counterparts from nonCS hermaphrodite

245 parents. Furthermore, preventing lipid reallocation by impairment of vitellogenesis in

246 either vit-2(If) or vit-5(If) was able to substantially ablate this effect, suggesting that the

247 improved survival is attributable specifically to lipid investment in the F1 generation (Fig.

248 7b-c). Altogether, it appears that while preventing embryonic lipid endowment may

249 promote adult survival post-CS, offspring that go on to experience future inclement

250 conditions suffer diminished survival rates, underscoring the evolutionary advantage of

251 terminal investment as a reproductive strategy. 

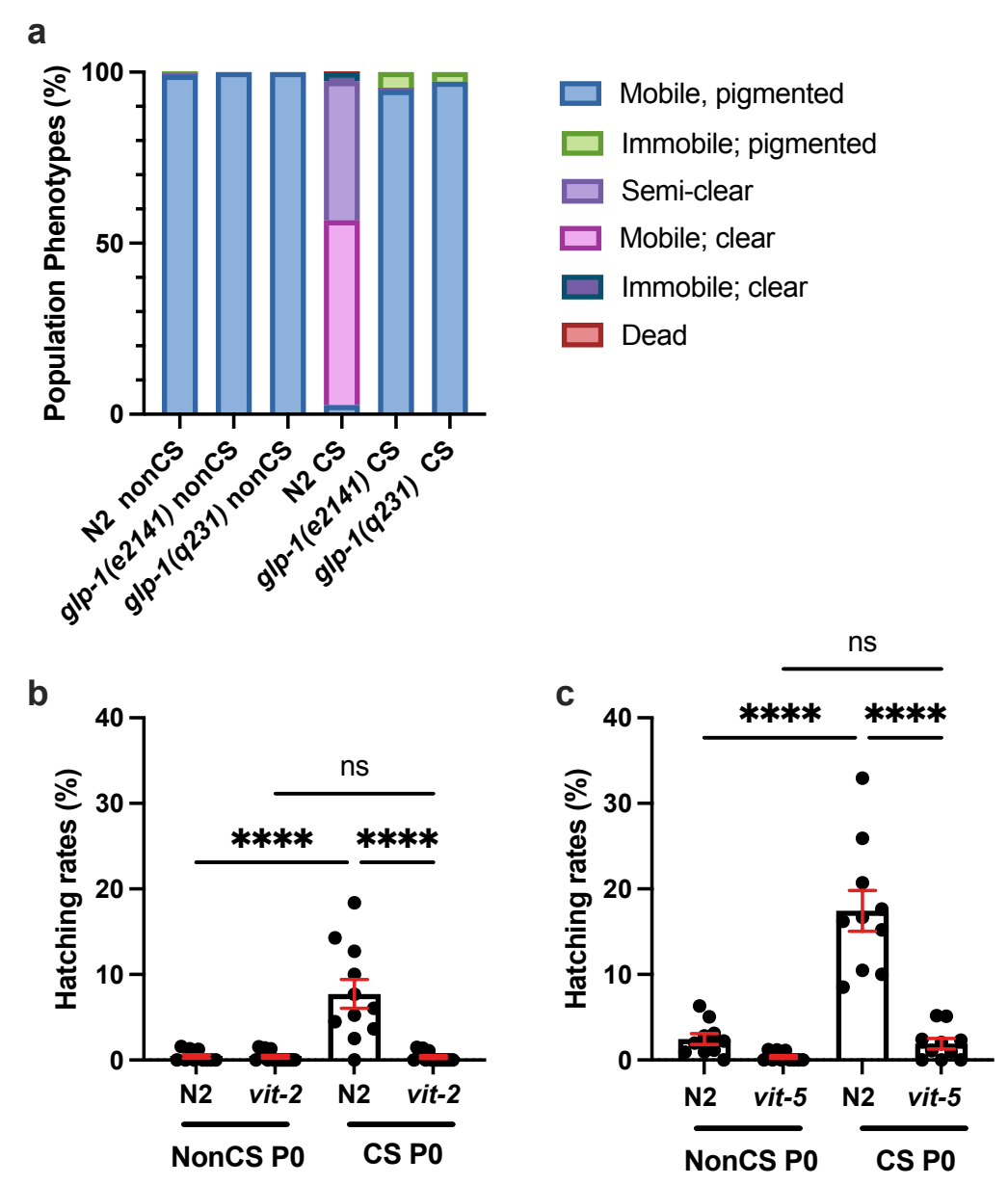

Figure 7. Cold shock response is a form of terminal investment. (a) $g l p-1$ (e2141) and $g l p-1$ (q231) were grown at the restrictive temperature $\left(25^{\circ} \mathrm{C}\right)$ to induce sterility and then habituated at $20^{\circ} \mathrm{C}$ for $4 \mathrm{~h}$. Worms were then cold shocked or mock shocked (nonCS) and recovered at $20^{\circ} \mathrm{C}$ for $24 \mathrm{~h}$ and phenotypes were scored ( $\mathrm{n} \geq 197$ worms for all conditions). (b) Young adult hermaphrodite N2 and vit-2(ok3211) (P0) were cold shocked or mock shocked (nonCS) for $4 \mathrm{~h}$ and recovered for $16 \mathrm{~h}$. Embryos from P0 treatments were collected from 16-18 $\mathrm{h}$ and cold shocked for $24 \mathrm{~h}$. Embryos were allowed to recover $24 \mathrm{~h}$ at $20^{\circ} \mathrm{C}$ and then the number of hatched embryos was quantified. Data points represent rates as percent hatched for a plate containing 50-100 embryos ( $\mathrm{n} \geq 10$ plates per condition; 2-way ANOVA $(\mathrm{F}(3,32)=20.87, \mathrm{P}<0.0001)$ with Tukey multiple comparison test $\left({ }^{* * * *} \mathrm{P}<0.0001\right)$. Bars are mean \pm s.e.m. (c) Performed as in $\mathbf{b}$ with $\mathrm{N} 2$ and vit-5 (ok3239) worms $(\mathrm{n}=10$ plates per condition; 2-way ANOVA $(\mathrm{F}(3,27)=35.28, \mathrm{P}<0.0001)$ and Tukey's multiple comparison test $\left({ }^{* * * *} \mathrm{P}<0.0001\right)$.

\section{Discussion}


256 We have provided here the first evidence that C. elegans, upon acute cold shock (CS), is able

257 to improve the success of progeny survival during future cold exposure by mobilizing lipids

258 to the germline as reproductive provisioning. This process seems to depend on neuronal

259 sensation of the cold temperature, which permits a shift of lipid localization from the soma

260 to the germline. Lipid reallocation is mediated by vitellogenins, and despite an overall

261 decrease in embryo output, the embryos in these worms are more resilient to future cold

262 exposure and display more robust hatching. The switch to a sudden last reproductive event

263 and resulting parental mortality is consistent with the adoption of a more semelparous

264 lifestyle [28]. In this instance, potentiation by a severe environmental stress to induce a

265 higher quality (rather than quantity) final reproductive investment suggests that C. elegans

266 has evolved a terminal investment response to deal with acute cold exposure.

267 Though, to our knowledge, no previous study has linked terminal investment to C. elegans,

268 there are other documented phenomena characterized by a similar trade off. For example,

269 the "age-dependent somatic depletion of fat (ASDF)" phenotype is characterized by age-

270 dependent changes in lipid homeostasis that are induced during both starvation and

271 oxidative stress and are highly reminiscent of post-cold-shock reallocation of lipids from

272 the intestine to the germline [24]. As is also the case with cold-shock-induced terminal

273 investment, reduction of vitellogenic transcripts (including vit-2) suppresses lipid

274 mobilization. Lipid reallocation during ASDF does appear to negatively impact parental

275 survival, but it is unclear whether this phenotype also holds functional significance for

276 offspring survival. Surprisingly, while the process governing the ASDF phenotype and cold

277 shock recovery are both regulated by the master stress response transcription factor SKN-

278 1/Nrf2, activating SKN-1 has opposite effects. When measuring ASDF, skn-1(gf) stimulates 
279 the shift of lipids from the intestine to the germline [24]; in contrast, skn-1(gf) prevents

280 lipid mobilization and promotes intestinal lipid sequestration following cold shock. It is

281 possible that the role of SKN-1 varies with age since ASDF occurs in much older adults than

282 those analyzed following cold stress. Although regulation may be via distinct pathways, the

283 existence of mechanistically similar lipid homeostasis responses to starvation, oxidative

284 stress, and cold stress suggests that terminal investment may be a more generalized

285 response to severe stressors in C. elegans.

286 Despite the fact that terminal investment requires extremely high parental resource

287 investment, there are other similar and less severe forms of maternal provisioning in $C$.

288 elegans. For example, osmotic stress shifts embryonic contents to include less glycogen and

289 more glycerol, a cryoprotectant [5]. It is particularly relevant to our study that mild

290 nutrient deprivation in hermaphrodites leads to intergenerational plasticity in which

291 embryos tend to be slightly larger and offspring are somewhat protected from the effects of

292 larval starvation [4]. Remarkably, this corresponds to decreased maternal insulin signaling

293 and upregulated vitellogenin provisioning in the germline, much as we report during cold

294 exposure [29]. It will be interesting in future experiments to dissect how thermosensory

295 signaling may hyperactivate vitellogenesis upon acute cold stress, especially as it relates to

296 different vitellogenin yolk protein products. Additionally, analyzing how various stressors

297 impact and modify embryonic composition will be important for understanding the extent

298 to which maternal investments are conserved. Taken together, it is clear that stressors in

299 the parental generation can have remarkable consequences on reproductive and survival

300 tradeoffs as well as offspring physiology and plasticity. 
301 Terminal investment in response to cold may have evolved in regularly fluctuating environmental conditions

303 Since the preeminent goal of survival is reproduction, terminal investment is sensible from

304 a fitness standpoint. But why would cold shock elicit this phenotype? Experimental

305 evolution in C. elegans reveals that deterministic maternal effects (those that attempt to

306 successfully invest offspring for survival in a particular environment) evolve in response to

307 predictably fluctuating environments [30]. Thus, we conjecture that predictably

308 encountered cold environments, such as those during seasonal freeze-thaw cycles in

309 temperate regions, are likely to have given rise to the reallocation phenotype. This could

310 happen by one of the two possible mechanisms. First, cold shock-induced damage may

311 signal to the worm that death is imminent and reproductive timespan is limited. Worms

312 may then funnel all their resources into embryos which are then better capable of surviving

313 cold stress. However, this seems unlikely since these data suggest that $C$. elegans that avoid

314 reallocation (or are genetically prevented from doing so) survive CS extremely well, which

315 is inconsistent with CS causing large amounts of damage. It is possible that these worms

316 are simply more resistant to CS-induced damage or are better able to repair damage in the

317 absence of reallocation, though this would need to be tested more rigorously.

318 A second, more plausible scenario is that seasonal cold cycles signal a coming winter

319 season with a low chance of offspring survival. At specific larval stages, worms can enter a

320 highly stress-resistant state (called the dauer stage) which may enable them to survive the

321 winter season and resume development when conditions are more favorable. If the better-

322 provisioned embryos of worms that have recently experienced a cold cycle survive 
323 successive cold shock, this could favor a larger percentage of offspring that are able to

324 successfully enter the dauer state. Long-term, this would translate to a higher probability

325 that offspring survive to reproduce when winter conditions are alleviated, continuing the

326 parent's lineage. This model accounts for the selection of the reallocation trait in

327 evolutionary history. It further suggests that rather than being induced as a result of

328 damage that signals impending death (and thus a final chance to reproduce), reallocation

329 may instead be prompted by a more general prediction of future damage to both the parent

330 and the offspring. Further experimentation is needed to assess this model and to determine

331 how it would fit into the known framework for terminal investment strategies.

332 Although we have largely considered simple maternal effects involving cytosolic

333 investments in this work, there is also the potential for epigenetic effects at the level of

334 gene expression to play a role in intergenerational and transgenerational offspring

335 survival. Indeed, the progeny of $C$. elegans submitted to various stressors demonstrate

336 improvements in stress survival to the same and other types of stress. Recently, a study by

337 Burton et al. [7] examined multigenerational responses to a number of stressors including

338 nutrient deprivation and pathogenic stress and found that multiple Caenorhabditis species

339 regulate gene expression in the F1 generation in a stress-specific manner to benefit

340 survival upon exposure to the same. These studies highlight the evolutionary advantage of

341 provisioning offspring for what is perceived to be a likely future. It would be interesting to

342 extend analyses of multigenerational gene regulation to see if this is also at play in the

343 context of CS.

\section{Terminal investment has consequences for population-level dynamics}


345 Terminal investment occurs across a broad phylogenetic range in response to many

346 stressors, and the potential ecological and evolutionary implications are serious in today's

347 global climate. Pathogen exposure commonly elicits terminal investment; one particularly

348 relevant example occurs in several species of frog in response to infection by

349 Batrachochytrium dendrobatidis (Bd), the perpetrator of severe global amphibian

350 declines. Rather than retaining resources for survival, individuals in many of these species

351 direct energy to reproduction, increasing gamete output. While producing more offspring

352 improves the likelihood of population persistence, this mode of reproduction does not

353 favor survival of frogs past infection, whereupon animals selected by resilience would

354 engender less susceptible offspring and gradually allow population resistance to the fungus

355 to evolve [31]. Thus, terminal investment poses a serious long-term survival risk for some

356 frog species faced with extinction from Bd. As global climates shift, it is unclear how

357 population dynamics will be affected by changing temperatures. Since terminal investment

358 is suspected to be a means of population persistence, it may play an important role in

359 continued population survival of various species.

360 In most documented cases, terminal investment results in increases to brood size but some

361 examples exist in which offspring quality is favored, such as in the tsetse fly, where stress

362 levels are positively correlated with the percent of body fat that is dedicated to offspring

363 [32]. The majority of terminal investment studies currently come from ecological studies

364 in non-model systems; thus, an understanding of the cellular components and mechanisms

365 involved in terminal investment, particularly in relation to quality investment, remains

366 largely elusive. Here we have identified a new terminal investment process in response to

367 cold stress in a genetically tractable and environmentally relevant model. The opportunity 
368 to better understand how this phenomenon is elicited and executed on the molecular scale

369 is an exciting prospect for future studies. With a more comprehensive understanding of the

370 impacts of stress on not only the parental generation but multiple generations thereafter,

371 we can better predict both an organism's physiology and population dynamics in response

372 to specific environmental factors.

\section{Materials and Methods}

\section{Strains and maintenance}

376 All strains were maintained on Nematode Growth Medium (NGM) seeded with Escherichia

377 coli $\mathrm{OP} 50$, at $20^{\circ} \mathrm{C}$ unless otherwise noted [33]. Worms were well-fed for at least three

378 generations before any experiments. Some strains were provided by the CGC, which is

379 funded by NIH Office of Research Infrastructure Programs (P40 OD010440). CGC strains

380 used in this study were N2 (Bristol) wild-type, SJ4005 zcIs4[hsp-4::gfp], BR5514 tax-

381 2(p671); tax-4(p678), GR2245 skn-1(mg570), RB2365 vit-2(ok3211), RB2382 vit-5(ok3239),

382 CB4037 glp-1(e2141), and JK509 glp-1(q231). JRP1036 skn-1(lax188) was generated from

383 SPC168 dvIs19; skn-1(lax188).

Cold shock survival

386 For adult cold shock, approximately 20-70 young adult worms (not yet gravid) were picked 387 to 0 P50-seeded $3.5 \mathrm{~cm} \mathrm{NGM}$ and placed at $2^{\circ} \mathrm{C}$ for 4 hours. After 4 hours cold shock, plates 388 were transferred to $20^{\circ} \mathrm{C}$ for worm recovery. Worms were scored at $1,4,24,48,72$, and 96 389 hours post-cold shock for survival and qualitative phenotypic assessments. A worm was 
considered dead when nose tap did not elicit any movement. Clear worms were

391 determined by a lack of almost all intestinal pigmentation, and immobile worms by a nose

392 tap that elicited only movement in the head region.

393

394 For embryonic cold shock, parents were cold shocked as above, allowed to recover for 15

395 hours, then transferred to fresh plates to lay embryos for 2 hours. This time window is the

396 peak of lipid reallocation following cold shock. Approximately 50-100 embryos were

397 transferred to very lightly seeded $3.5 \mathrm{~cm} \mathrm{NGM}$ plates and cold shocked at $2^{\circ} \mathrm{C}$ for 24

398 hours. Following a 24-hour recovery at $20^{\circ} \mathrm{C}$, the number of hatched and unhatched

399 embryos were counted. Embryos were considered hatched if the entirety of the L1 larva

400 was visible in a non-curled state, and the larva was not dead.

401 During all cold shock experiments, plates were placed directly on the incubator shelf in a

402 monolayer rather than a stack both during cold shock and the early stages of recovery to

403 facilitate uniform temperature changes among plates.

\section{C. elegans Fluorescence Imaging and Quantification}

406 zcIs4[hsp-4::GFP] worms were either heat shocked at $35^{\circ} \mathrm{C}$ for $2 \mathrm{~h}$, cold shocked according

407 to standard protocol, or non-shocked. After $12 \mathrm{~h}$ recovery at $20^{\circ} \mathrm{C}$, worms were picked to a

408 drop of M9 containing 5 mM sodium azide. Worms were imaged on a Nikon Eclipse 90i

409 microscope at exposure times of $2.9 \mathrm{~ms}$ for DIC and $900 \mathrm{~ms}$ for GFP.

411 The fixed Nile Red staining protocol was modified from Pino et al. [34]. Briefly, at $12 \mathrm{~h}$ post-

412 cold shock, 50-75 worms were washed once with M9 and fixed for three minutes at room 


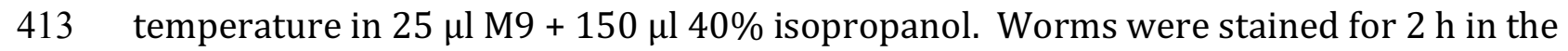

414 dark with gentle rocking in $175 \mu \mathrm{l} 40 \%$ isopropanol containing $0.6 \%$ Nile Red stock $(0.5$

$415 \mathrm{mg} / \mathrm{ml}$ in acetone). Samples were washed with M9 for $30 \mathrm{~min}$ in the dark with gentle

416 rocking, then mounted on a 2\% agarose pad for imaging. Worms were imaged on a Nikon

417 Eclipse 90i microscope at exposure times of $3 \mathrm{~ms}$ for DIC and $400 \mathrm{~ms}$ for GFP (figures 1, 3,

4184,5 ) or on a Zeiss Axio Imager Z1 microscope with exposure times of 3 ms for DIC and 500

419 ms for GFP (figures 2, 6).

420 Scale bars $(100 \mu \mathrm{m})$ were added and all images were rotated and cropped using ImageJ. No

421 other image manipulations were performed.

422

423 Images of fluorescent worms taken on the Nikon Eclipse 90i microscope (figures 1, 3, 4, 5)

424 were analyzed using NIS Elements Data Analysis software; images taken on the Zeiss Axio

425 Imager Z1 microscope (figures 2, 6) were analyzed using ImageJ. In either case, total

426 fluorescence was determined by outlining the entire worm as a Region of Interest (ROI)

427 and calculating the average fluorescence for that ROI. The number of internal embryos and

428 the number of fluorescent embryos were determined by visual counts.

$430 \quad$ Statistical analysis

431 All data represent at least three independent replicates. Statistical analysis was performed

432 in GraphPad Prism 9 with an alpha value of $\mathrm{P}<0.05$. For samples with 2 conditions,

433 nonparametric Mann-Whitney U tests were performed and reported with two-tailed P

434 values. In cases with greater than 2 conditions, Kruskal-Wallis nonparametric ANOVA were

435 performed with Dunn's Multiple Comparison test. For hatching analyses, a Grubb's test was 
436 conducted through GraphPad to identify outliers in the data, with one point identified as an

437 outlier $(\mathrm{Z}=3.4005$, two-tailed $\mathrm{P}<0.01)$ and removed. d'Agostino \& Pearson normality tests

438 were then applied to the remaining values and normal distribution was confirmed by a P >

4390.05 for each condition. After validating data normality, 2-way ANOVAs were used to

440 compare the mean of each condition with a Tukey's Multiple Comparison test.

\section{Acknowledgements}

442 The authors thank Sean Curran, Ralph Baumeister, and Thomas Heimbucher for reagents

443 and useful discussions. Some strains were provided by the CGC, which is funded by NIH

444 Office of Research Infrastructure Programs (P40 OD010440). Funding was provided by

445 Gettysburg College.

\section{Author Contributions}

447 LG and JRP conceived and performed experiments, analyzed and interpreted data, and 448 prepared the manuscript.

\section{Competing interests}

450 The authors declare no competing interests.

\section{References}

452 1. Duffield, K. R., Bowers, K. E., Sakaluk, S. K. \& Sadd, B. M. A dynamic threshold model

453 for terminal investment. Behav. Ecol. Sociobiol. 71, (2017).

454 2. Gulyas, L. \& Powell, J. R. Predicting the Future: Parental Progeny Investment in

455 Response to Environmental Stress Cues. Front. cell Dev. Biol. 7, (2019).

456 3. Herman, J. J. \& Sultan, S. E. Adaptive Transgenerational Plasticity in Plants: Case 
Studies, Mechanisms, and Implications for Natural Populations. Front. Plant Sci. 0, 102 (2011).

459 4. Hibshman, J. D., Hung, A. \& Baugh, L. . R. Maternal Diet and Insulin-Like Signaling Control Intergenerational Plasticity of Progeny Size and Starvation Resistance. PLoS Genet. 12, (2016).

462 5. Frazier, H. N. \& Roth, M. B. Adaptive Sugar Provisioning Controls Survival of $C$. elegans Embryos in Adverse Environments. Curr. Biol. 19, 859-863 (2009).

6. Burton, N. O. et al. Cysteine synthases CYSL-1 and CYSL-2 mediate C. elegans

7. Burton, N. O. et al. Intergenerational adaptations to stress are evolutionarily conserved, stress-specific, and have deleterious trade-offs. bioRxiv Drosophila as a general stress response to cold temperatures. J. Insect Physiol. 107, 2021.05.07.443118 (2021) doi:10.1101/2021.05.07.443118.

9. Sun, W. et al. Cold-induced epigenetic programming of the sperm enhances brown

8. Lirakis, M., Dolezal, M. \& Schötterer, C. Redefining reproductive dormancy in

474 10. Garrity, P. A., Goodman, M. B., Samuel, A. D. \& Sengupta, P. Running hot and cold: behavioral strategies, neural circuits, and the molecular machinery for thermotaxis

477 11. Ohta, A., Ujisawa, T., Sonoda, S. \& Kuhara, A. Light and pheromone-sensing neurons regulates cold habituation through insulin signalling in Caenorhabditis elegans. Nat. 
480 12. Takeishi, A., Takagaki, N. \& Kuhara, A. Temperature signaling underlying thermotaxis and cold tolerance in Caenorhabditis elegans. doi:10.1080/01677063.2020.1734001.

13. Sonoda, S., Ohta, A., Maruo, A., Ujisawa, T. \& Kuhara, A. Sperm Affects Head Sensory Neuron in Temperature Tolerance of Caenorhabditis elegans. Cell Rep. 16, 56-65 (2016).

14. Jiang, W. et al. A genetic program mediates cold-warming response and promotes stress-induced phenoptosis in C. elegans. Elife 7, (2018).

15. Robinson, J. D. \& Powell, J. R. Long-term recovery from acute cold shock in Caenorhabditis elegans. BMC Cell Biol. 17, 1-11 (2016).

16. McGhee, J. D. The C. elegans intestine. WormBook 1-36 (2007) doi:10.1895/WORMBOOK.1.133.1.

491 17. Greenspan, P., Mayer, E. P. \& Fowler, S. D. Nile red: a selective fluorescent stain for intracellular lipid droplets. J. Cell Biol. 100, 965-973 (1985).

18. Colinet, H., Lee, S. F. \& Hoffmann, A. Temporal expression of heat shock genes during cold stress and recovery from chill coma in adult Drosophila melanogaster. FEBS J.

496 19. Coburn, C. M. \& Bargmann, C. I. A Putative Cyclic Nucleotide-Gated Channel Is (1996).

499 20. Komatsu, H., Mori, I., Rhee, J. S., Akaike, N. \& Ohshima, Y. Mutations in a Cyclic $500 \quad$ Nucleotide-Gated Channel Lead to Abnormal Thermosensation and Chemosensation in C. elegans. Neuron 17, 707-718 (1996). 
Generated Reactive Oxygen Species Trigger Protective SKN-1 Activity via p38 MAPK

Signaling during Infection in C. elegans. PLOS Pathog. 7, e1002453 (2011).

505

22. Papp, D., Csermely, P. \& Sőti, C. A Role for SKN-1/Nrf in Pathogen Resistance and Immunosenescence in Caenorhabditis elegans. PLOS Pathog. 8, e1002673 (2012).

23. Dodd, W. et al. A Damage Sensor Associated with the Cuticle Coordinates Three Core (2018).

24. Lynn, D. A. et al. Omega-3 and - 6 fatty acids allocate somatic and germline lipids to ensure fitness during nutrient and oxidative stress in Caenorhabditis elegans. Proc. Natl. Acad. Sci. U. S. A. 112, 15378-15383 (2015).

513 25. Lerhbach, N. J. \& Ruvkun, G. Proteasome dysfunction triggers activation of SKN1A/Nrf1 by the aspartic protease DDI-1. Elife $\mathbf{5},(2016)$.

515 26. Perez, M. F. \& Lehner, B. Vitellogenins - Yolk Gene Function and Regulation in Caenorhabditis elegans. Front. Physiol. 0, 1067 (2019).

517 27. Spieth, J. \& Blumenthal, T. The Caenorhabditis elegans vitellogenin gene family includes a gene encoding a distantly related protein. Mol. Cell. Biol. 5, 2495-2501 (1985).

520 28. Hughes, P. W. Between semelparity and iteroparity: Empirical evidence for a $521 \quad$ continuum of modes of parity. Ecol. Evol. 7, 8232-8261 (2017).

522 29. Jordan, J. M. et al. Insulin/IGF Signaling and Vitellogenin Provisioning Mediate Intergenerational Adaptation to Nutrient Stress. Curr. Biol. 29, 2380-2388.e5 (2019).

524 30. Proulx, S. R. \& Teotónio, H. What Kind of Maternal Effects Can Be Selected For in Fluctuating Environments? Am. Nat. 189, E118-E137 (2017). 
526 31. Brannelly, L. A., Webb, R., Skerratt, L. F. \& Berger, L. Amphibians with infectious disease increase their reproductive effort: evidence for the terminal investment hypothesis. Open Biol. 6, (2016).

529 32. Hargrove, J. W., Muzari, M. O. \& English, S. How maternal investment varies with environmental factors and the age and physiological state of wild tsetse Glossina pallidipes and Glossina morsitans morsitans. R. Soc. open Sci. 5, (2018).

532 33. Brenner, S. The genetics of Caenorhabditis elegans. Genetics 77, 71-94 (1974).

533 34. Pino, E. C., Webster, C. M., Carr, C. E. \& Soukas, A. A. Biochemical and high throughput 534 microscopic assessment of fat mass in Caenorhabditis elegans. J. Vis. Exp. (2013) doi:10.3791/50180. 